\title{
TAFONOMÍA DE MICROVERTEBRADOS EXHUMADOS DEL SITIO ARQUEOLÓGICO AGUA DE LOS CABALLOS-1 (MENDOZA, ARGENTINA)
}

\section{TAPHONOMY OF MICROVERTEBRATES RECOVERED FROM AGUA DE LOS CABALLOS ARCHAEOLOGICAL SITE (MENDOZA, ARGENTINA)}

\author{
Fernando Julián Fernández \\ CONICET. Cátedra de Anatomía Comparada, Facultad de Ciencias Naturales y Museo, UNLP, \\ E-mail: fernandezf77@yahoo.com.ar
}

Presentado el: 15/10/2012 - Aceptado 11/11/2012

\section{Introducción}

El sitio arqueológico Agua de los Caballos-1(ACA-1), es un alero rocoso que se localiza en el extremo norte del campo volcánico de La Payunia, Mendoza, sobre la margen izquierda del arroyo Agua de los Caballos ( $35^{\circ} 22^{\prime} 03^{\prime \prime}$ S, 68 $68^{\circ} 07^{\prime \prime}$ O; 1025 m s.n.m.; Figura 1). Desde una perspectiva fitogeográfica, ACA-1 se encuentra emplazado en la provincia del Monte (Cabrera 1976). El alero presenta la abertura hacia el este y tiene una superficie de 6,5 m2. Durante enero de 1996, un equipo de arqueólogos dirigidos por Adolfo Gil excavó una cuadrícula de $2 \mathrm{~m} 2$, donde se extrajeron 17 niveles artificiales de $5 \mathrm{~cm}$, hasta alcanzar la roca madre (Gil 2006).

Gil (2006), utilizando criterios cronológicos, tecnológicos y densidad de materiales arqueológicos, dividió la secuencia de ACA-1 en dos componentes culturales. El Componente I (niveles 0 a 6), cronológicamente posterior a ca. 250 años C14 AP, y el Componente II (niveles 7 a 17), comprendido entre ca. 1200 años C14 AP y ca. 250 años C14 AP. En términos generales, Gil (2006) postuló que ACA-1 corresponde a un sitio de actividades múltiples, donde se realizaron en forma casi continua tareas de talla, reacondicionamiento de instrumentos, procesamiento de animales, recolección vegetal y molienda y uso ritual, entre otras; produciéndose algunos cambios del registro en los últimos 250 años C14 AP (véase Gil 2006). 


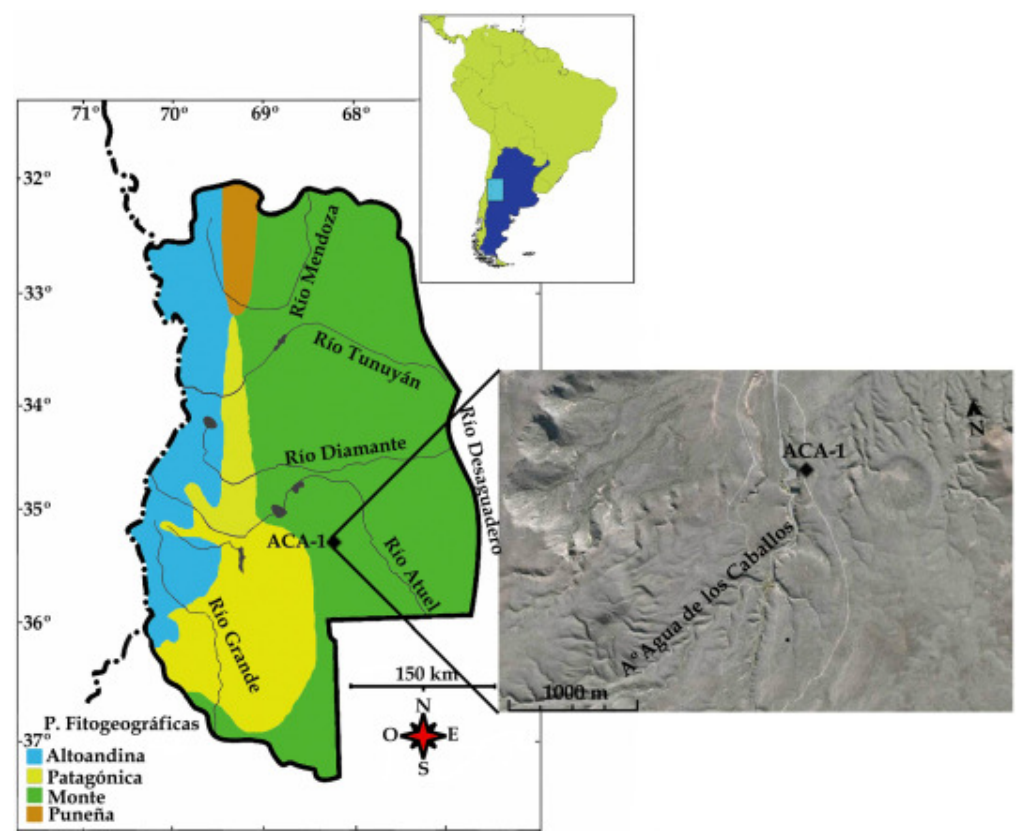

Figura 1. Mapa de Mendoza con las unidades fitogeográficas (sensu Cabrera 1976), mostrando la ubicación del sitio arqueológico ACA-1.

En este trabajo se presentan los resultados del estudio taxonómico y tafonómico, realizado sobre los conjuntos de microvertebrados $(<1 \mathrm{~kg})$ recuperados del sitio ACA-1, discutiendo sus implicancias en el marco de su contexto arqueológico regional.

\section{Materiales y Métodos}

Los microvertebrados se recuperaron en zaranda de $2 \mathrm{~mm}$ de malla y las determinaciones taxonómicas se realizaron utilizando materiales de comparación y fuentes bibliográficas (e.g., Fernández et al. 2011). Asimismo, se calculó el Número de Especímenes Identificados por Taxón (NISP), Número Mínimo de Elementos (MNE) y Número Mínimo de Individuos (MNI) (cf. Grayson 1984).

Para evaluar el origen de las acumulaciones de microvertebrados, se siguió la metodología propuesta por Andrews (1990). En este marco, se observaron las marcas de digestión gástrica, grado de fractura y la abundancia relativa de los elementos esqueletales (cf. Andrews 1990). También se evaluaron variables antrópicas tales como marcas de corte y patrones de termoalteración (cf. Medina et al. 2012) y variables postdepositacionales tales como meteorización, pisoteo, marcas de raíces, impresiones de óxido de manganeso, abrasión por transporte hídrico y corrosión sedimentaria (cf. Andrews 1990; Fernández-Jalvo y Andrews 2003; Korth 1979).

\section{Resultados}

Se analizaron 97 especímenes, 26 correspondientes al Componente I y 71 al Componente II (Tabla 1). Ambos componentes están dominados por micromamíferos. Entre ellos, igualmente representados se encuentran los roedores histricognatos Galea leucoblephara 


\begin{tabular}{|c|c|c|c|c|c|c|}
\hline \multirow{2}{*}{$\begin{array}{l}\text { Taxones/ Procedencia } \\
\text { Amphibia/ Anura indet. }\end{array}$} & \multicolumn{3}{|c|}{$\begin{array}{l}\text { Componente I } \\
\text { NISP MNE MNI }\end{array}$} & \multicolumn{3}{|c|}{$\begin{array}{l}\text { Componentes II } \\
\text { NISP MNE MNI }\end{array}$} \\
\hline & & & & 1 & 1 & 1 \\
\hline Reptilia / Sauria indet. & & & & 1 & 1 & 1 \\
\hline Aves indet. & 1 & 1 & 1 & & & \\
\hline Passeriformes / Oscinae indet. & 1 & 1 & 1 & & & \\
\hline \multicolumn{7}{|l|}{ Columbiformes } \\
\hline Zenaida auriculata & 1 & 1 & 1 & & & \\
\hline Mammalia / Rodentia indet. & 3 & 3 & 1 & 20 & 16 & 1 \\
\hline \multicolumn{7}{|l|}{ Ctenomyidae } \\
\hline Ctenomys sp. & & & & 2 & 2 & 1 \\
\hline Caviidae indet. & 10 & 10 & 2 & 24 & 23 & 2 \\
\hline Microcavia australis & & & & 1 & 1 & 1 \\
\hline Galea leucoblephara & & & & 1 & 1 & 1 \\
\hline Cricetidae/ Sigmodontinae indet. & 9 & 9 & 2 & 19 & 19 & 2 \\
\hline Phyllotis xanthopygus & & & & 1 & 1 & 1 \\
\hline Graomys griseoflavus & 1 & 1 & 1 & & & \\
\hline Eligmodontia sp. & & & & 1 & 1 & 1 \\
\hline Total & 26 & 26 & 9 & 71 & 66 & 12 \\
\hline
\end{tabular}

Tabla 1. Composición taxonómica de los conjuntos de microvertebrados de ACA-1 (expresada en NISP, MNE y MNI).

(cuis común), Microcavia australis (cuis chico) y Ctenomys sp. (tuco-tucos) y los roedores sigmodontinos Graomys griseoflavus (pericote común), Phyllotis xanthopygus (pericote panza gris) y Eligmodontia sp. (lauchas colilargas). Con proporciones menores se encuentran un saurio, un anuro, el colúmbido Zenaida auriculata (torcaza) y un paseriforme.

El 31,2\% de todos los especímenes exhumados de ACA-1 sufrieron corrosión digestiva, concentrándose en la categoría ligera $(27,1 \%)$ y en algunos casos en las categorías moderada $(3,1 \%)$ y fuerte (1\%). Precisamente en el Componente I la corrosión fue del 19,2\% y en el Componente II alcanzó el 33,8\% del total. En el Componente I no se registraron dientes con evidencias de digestión, aunque sí elementos postcraneales (Figura $2 \mathrm{~A}$ ). En el Componente II el $52,6 \%$ de los restos dentarios estaban digeridos de forma ligera y el $58,3 \%$ de los elementos postcraneales presentaron corrosión principalmente en la categoría ligera $(41,7 \%)$ y en menor medida en la categoría moderada (16,6\%) (Figura 2 B).

El promedio de la abundancia relativa de los elementos esqueletales fue baja (Componente I = 9,8\%; Componente II = 12,8\%). En los dos componentes se registró un patrón similar de representación de partes esqueletales, donde los elementos más abundantes fueron los maxilares, pelvis, fémures y tibias. 


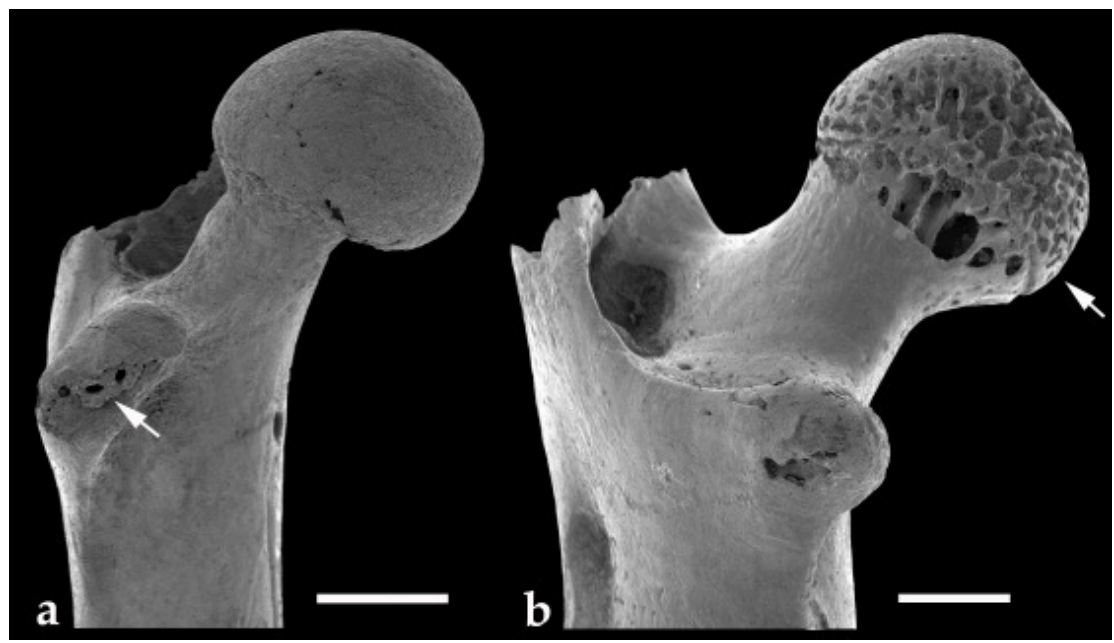

Figura 2. Elementos postcraneales con evidencias de corrosión digestiva: a) fémur de Sigmodontinae con corrosión ligera (Componente I, Nivel 6); b) fémur de Caviinae con corrosión moderada (Componente II, Nivel 14). Escalas: $1 \mathrm{~mm}$.

El 93,7\% de todos los especímenes recuperados de ACA-1 estaban fracturados (Componente I = 96,1\%; Componente II = 91,5\%). No se recuperaron cráneos completos; correspondiendo a maxilares sin arcos zigomáticos y a fragmentos de premaxilares. En ambos componentes se registraron ca. del $50 \%$ de pérdidas dentarias. Las mandíbulas se encontraron muy fragmentadas, recuperándose una sola completa en el Componente II. La mayoría de los molares e incisivos se hallaron enteros. Sólo el 25\% de los húmeros, fémures y tibias estaban completos. Los metapodios y falanges estaban enteros y se registró una vértebra fracturada. No obstante, los elementos más frágiles como las cinturas se encontraron fragmentados. Además, un $80 \%$ de los elementos postcraneales rotos recuperados de ambos componentes exhibieron superficies de fracturas con ángulos agudos y bordes ásperos. Por otra parte, no se encontraron esqueletos articulados de Ctenomys sp., que pudiera explicar una muerte natural en el sitio.

Por último, no se registraron restos termoalterados ni con marcas de corte. Un bajo porcentaje exhibieron evidencias de meteorización (Componente I $=11,5 \%$; Componente II $=12,7 \%$ ). Se registró un resto óseo con marcas de raíces (i.e., Sphenoichnia) en el Componente II. No se recuperaron especímenes con impresiones de óxido de manganeso, ni evidencias de redondeamiento en los extremos de los huesos. No obstante, el 5,2\% de todos los restos recuperados de ACA-1 mostró evidencias de corrosión sedimentaria, siendo el Componente I $(11,5 \%)$ más afectado que el Componente II $(2,8 \%)$.

\section{Discusión}

La presencia de G. griseoflavus en el Componente I (ca. 250 años C14 AP), de G. leucoblephara y M. australis en el Componente II (ca. 1200-250 años C14 AP) sugiere que los conjuntos de micromamíferos del sitio ACA-1, están formados por especies que habitan principalmente en el desierto del Monte. Asimismo, con el agregado de elementos ampliamente distribuidos en Patagonia y frecuentes en ambientes xéricos, vinculados a estepas arbustivas abiertas con afloramientos rocosos en el Componente II (P. xanthopygus y Eligmodontia sp.). A pesar de 
ser una muestra escasa, se observa que coincide con las comunidades de micromamíferos actuales cercanas al sitio (Fernández 2010, 2012), indicando una estabilidad taxonómica y ambiental. Este escenario es posterior a un período de transición con la estepa Patagónica, señalado en base al registro de micromamíferos proveniente de un sitio arqueológico cercano a ACA-1 (Agua de La Mula) entre ca. 1600 y 1000 años C14 AP (Fernández 2010, 2012) y en forma casi sincrónica con la ocupación humana efectiva de La Payunia (véase Gil 2006).

La ausencia de marcas de corte y de restos quemados, sugieren que los grupos humanos que habitaron el sitio, no participaron en la génesis de los conjuntos de microvertebrados. No obstante, las evidencias tafonómicas señalan que los conjuntos de microvertebrados tuvieron su origen principalmente por la actividad depredadora de aves Strigiformes. Esto se sustenta principalmente en base al registro de evidencias de corrosión digestiva, principalmente ligera, lo cual coincide con los estudios actualísticos realizados en base a egagrópilas de Strigiformes (Andrews 1990; Gómez 2007). Sin embargo, los altos niveles de fractura, pérdida dentaria y los patrones de abundancias relativas de partes esqueletales, no concuerdan exactamente con los valores obtenidos por Andrews (1990). Posiblemente, procesos postdepositacionales comunes en reparos, tales como el pisoteo pudieron alterar la composición original de los conjuntos. Esto se fundamenta en que los elementos más chicos se encontraron completos (i.e., vértebras, metapodios y falanges), en congruencia con estudios experimentales realizados por Andrews (1990).

Por otra parte, los conjuntos parecen no haber sido afectados o fueron escasamente afectados por otros tipos de procesos postdepositaciones como meteorización, acción de raíces, óxido de manganeso y abrasión por transporte hídrico (Andrews 1990; FernándezJalvo y Andrews 2003; Korth 1979), indicando buena preservación y rápido enterramiento, previamente a ser afectados por el pisoteo. Únicamente, como característica distintiva del contexto postdepositacional se encontraron algunos restos con evidencias de corrosión sedimentaria -mayoritariamente en el Componente I- posiblemente producto de la humedad del suelo (véase Andrews 1990).

Finalmente, hasta el momento no se han encontrado en La Payunia, conjuntos de microvertebrados con indudables evidencias tafonómicas asociadas a actividades antrópicas de consumo y/o explotación (Giardina 2010; Fernández 2012). Esto no coincide con el proceso de intensificación de los recursos planteado en esta área a partir de los 1000 años C14 AP (Gil 2006). Este proceso implicó un conjunto de estrategias, disparadas por un aumento demográfico y de territorialidad, que le permitieron a los cazadores-recolectores incorporar numerosos recursos alimenticios a su dieta, tales como plantas y vertebrados medianos (Giardina 2010; Gil 2006). No obstante, este proceso pudo no haber sido tan marcado como para que las poblaciones humanas tuviesen que explotar a los microvertebrados.

\section{Agradecimientos}

A mis directores de tesis doctoral Luciano De Santis y Gustavo Neme. A los revisores por sus valiosos comentarios. Al CONICET y la Facultad de Ciencias Naturales y Museo (UNLP).

\section{Bibliografía citada}

Andrews, P.

1990 Owls, caves and fossils. University of Chicago Press, Chicago. 
Cabrera, A.L.

1976 Regiones fitogeográficas argentinas. Enciclopedia Argentina de Agricultura y Jardinería 1:1-85. Fernández, F.J.

2010 Paleozoogeography of the wine mouse (Akodon oenos) \& late Holocene paleoenvironments in south-central of Mendoza, Argentina. Ethnobiology Letters 1: 52-57.

2012 Microvertebrados del Holoceno de sitios arqueológicos en el sur de Mendoza (República Argentina): aspectos tafonómicos y sus implicancias en la subsistencia humana. Tesis doctoral inédita. Facultad de Ciencias Naturales y Museo, UNLP, La Plata.

Fernández, F., F. Ballejo, G. Moreira, E. Tonni y L. De Santis

2011 Roedores cricétidos de la provincia de Mendoza. Guía cráneo-dentaria orientada para su aplicación en estudios zooarqueológicos. Editorial SAA y Universitas Sarmiento, Córdoba.

Fernández-Jalvo, Y. y P. Andrews

2003 Experimental effects of water abrasion on bone fragments. Journal of Taphonomy 1: 147-163.

Giardina, M.

2010 El aprovechamiento de la avifauna entre las sociedades cazadoras-recolectoras del Sur de Mendoza, un enfoque arqueozoológico. Tesis doctoral inédita. Facultad de Ciencias Naturales y Museo, UNLP, La Plata.

Gil, A.

2006 Arqueología de La Payunia (Mendoza, Argentina). El poblamiento humano en los márgenes de la agricultura. Bar International Series 1477, Oxford.

Gómez, G.N.

2007 Predators categorization based on taphonomic analysis of micromammals bone: a comparison to proposed models. Taphonomy and Zooarchaeology in Argentina (ed. por M.A Gutierrez, L. Miotti, G. Barrientos, G. Mendoni Goñalons y M. Salemme), pp. 1-16. BAR. International Series 1601. Oxford.

Grayson, D.K.

1984 Quantitative Zooarchaeology: topics in the analysis of archaeological faunas. Academics Press, Orlando, Florida.

Korth, W.

1979 Taphonomy of Microvertebrate Fossil Assemblages. Annals of Carnegie Museum 15: 235-285.

Medina, M., P. Teta y D. Rivero

2012 Burning damage and small-mammal human consumption in Quebrada del Real 1 (Córdoba). Journal of Archaeological Science 39: 737-743. 